\title{
Autoevaluación de fortalezas, debilidades y confianza de los médicos de atención primaria en el abordaje de enfermedades reumatológicas
}

\author{
Daniel Pacheco $\mathbf{R}^{\mathbf{1}}$, Héctor $\mathbf{G}$ atica $\mathbf{R}^{\mathbf{2}}$, Sonia Kaliski $\mathbf{K}^{\mathbf{3}}$. \\ Self assessment of strengths, \\ weaknesses and self confidence \\ of primary care physicians \\ taking care of rheumatic diseases
}

Background: Rheumatologic diseases are common and frequently managed by primary care physicians. Aim: To assess strengths, weaknesses and self confidence of primary care physicians in the management of rheumatic diseases. Material and methods: A self assessment and anonymous questionnaire was mailed to primary care physicians of two Chilean regions. Using a 10 points Likert scale, they were asked about personal interest, undergraduate training, continuous medical education, availability of medical literature, complementary laboratory tests and consultation with a rheumatologist. Medical skills, knowledge, therapeutic approach and performance of rheumatologic procedures were evaluated under the item confidence. Results: Three hundred forty seven out of 763 physicians (45\%) answered the questionnaire. Their age range extended from 25 to 75 years, $59 \%$ were male, $58 \%$ were Chilean and $74 \%$ worked in the Metropolitan region. The worst evaluated parameters were availability of literature with a score of $2.2 \pm 2$, access to consultation with a rheumatologist $(3.8 \pm 2.2)$ and to continuous medical education (4.3 \pm 2.7$)$. Physicians had a better confidence in their knowledge $(6.7 \pm 1.5)$ and in their therapeutic approach $(6.1 \pm 1.5)$. The worst confidence score was for shoulder injection therapy $(3.4 \pm 2.6)$. Continuous medical education correlated with knowledge but not with clinical skills. Conclusions: Primary care physicians perform a bad assessment of their skills in rheumatology. They have a low level of confidence in their clinical skills to perform rheumatologic procedures. Continuous medical education improves confidence in knowledge but not in skills (Rev Méd Chile 2006; 134: 813-20).

(Key words: Primary health care; Rheumatology; Self assessment (Psychology))

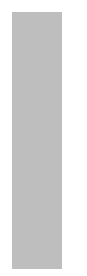

\footnotetext{
Recibido el 31 de marzo, 2005. Aceptado el 3 de enero, 2006.

Trabajo financiado por Education and Publication Committee. Pan American League of Associations for Rheumatology (PANLAR). No tuvo participación en el diseño ni análisis del estudio.

${ }^{1}$ Unidad de Reumatología e Inmunología, Hospital Clínico San Borja Arriarán, Campus Centro, Facultad de Medicina, Universidad de Chile. ${ }^{2}$ Unidad de Reumatología y Epidemiología Clínica, Hospital Clínico José J. Aguirre, Facultad de Medicina, Universidad de Chile. ${ }^{3}$ Unidad de Reumatología, Facultad de Medicina, Universidad de la Frontera, Temuco.
}

Correspondencia a: Dr. Daniel Pacheco R. Los Molinos 8309.

Vitacura, Santiago, Chile. Fono: 2016958. Fax: 2360224.

E mail: danielpacheco@mi.cl 
$\mathrm{U}$ na buena estrategia para mejorar la salud de la población, apoyada por la Organización Mundial de la Salud, es maximizar la cobertura de la atención ${ }^{1}$. Para lograrlo, se ha dado importancia a la Atención Primaria de Salud (APS). Uno de los roles principales del médico de Atención Primaria (MAP) es evaluar pacientes, constituyéndose en el primer eslabón de la red que puede llegar a involucrar a la atención especializada.

Las enfermedades reumatológicas (REUMA), definidas como las que comprometen el sistema músculo esquelético de cualquier etiología ${ }^{2}$, son causa frecuente de morbilidad, licencias, invalidez y requerimiento de los sistemas de salud ${ }^{3}$. Su impacto en APS es alto, ya que constituyen entre 9 y $20 \%$ de las visitas espontáneas en ese nivel ${ }^{4-6}$. Debido a esta gran demanda, es imposible que todas las consultas sean derivadas a un reumatólo$\mathrm{go}^{7}$, por lo que el MAP tiene que discriminar entre enfermedades que puede abordar de las que debe derivar.

Dado lo anterior, cabe preguntarse si las condiciones básicas de trabajo de los MAP en APS son adecuadas para el enfrentamiento de REUMA y también, indagar respecto al grado de confianza (Confianza) de los MAP en el manejo de estas enfermedades ${ }^{8}$.

La conducta de un médico, ante un problema clínico y las decisiones que tome, depende en gran medida de la percepción de su autoeficacia, es decir, el juicio personal respecto a su capacidad de realizar una actividad médica. Se puede determinar la percepción de autoeficacia midiendo Confianza del médico ante un desafío clínico ${ }^{9,10}$.

Condiciones de trabajo insuficientes y baja Confianza de los MAP para el abordaje de las enfermedades, podrían traducirse en un trabajo médico ineficaz ${ }^{8}$.

Los objetivos de este estudio fueron evaluar las fortalezas y debilidades de la APS para el abordaje de enfermedades reumatológicas, la autoevaluación de Confianza de los MAP en el abordaje clínico de estas enfermedades y su comparación con Confianza para enfrentar enfermedades distintas a las reumatológicas. Distintos aspectos biodemográficos, de educación de pre y postítulo y de condiciones asociadas a la práctica médica fueron evaluados adicionalmente para explorar su asociación con los hallazgos encontrados.

\section{MATERIAL Y MÉTODO}

Se diseñó una encuesta de autoevaluación para ser respondida en forma voluntaria, anónima e individual por MAP, que incluía tres temas de estudio: Datos demográficos e información general; evaluación de fortalezas y debilidades y Confianza respecto a REUMA frecuentes ${ }^{6}$ y otras enfermedades (Tabla 1).

La encuesta fue generada por reumatólogos de la Unidad de Reumatología del Hospital Clínico San Borja-Amiarán, revisada por especialistas en medicina familiar y sometido al análisis de un grupo de médicos del Hospital Clínico de la Universidad de Chile, ajustándose a la metodología convencional para este tipo de instrumento ${ }^{11-13}$. Se informó a los directores de cada centro respecto a la investigación, se obtuvo su autorización y se nombró un coordinador. El instrumento fue enviado por comeo a todos los MAP de los 116 consultorios urbanos y rurales de la región metropolitana y los 22 de la IX región, entre marzo y abril de 2001; cada MAP recibió un sobre con franqueo prepagado para el retomo de la encuesta antes de dos meses. Se coordinó telefónicamente para inducir el máximo de respuestas. La evaluación se efectuó utilizando una escala numérica tipo Likert, que es la forma de opción de respuesta para ítems unidimensionales de mayor uso en las ciencias sociales $^{14}$, de 1-10 puntos. Se incluyeron instrucciones precisas sobre el sentido de las opciones de respuesta, adecuando los descriptores verbales terminales a cada pregunta. Se fijó 1 punto a la evaluación menor y 10 puntos a la mayor. Ejemplo: «on la siguiente escala evalúe su grado de confianza para realizar una anamnesis reumatológica»:

Mín confianza 12345678910 Máx confianza

Estadística. La descripción de los datos se efectuó como promedio del puntaje para cada ítem, según la escala de opciones de respuesta numérica y su desviación estándar ( \pm DE). El análisis estadístico se realizó con pruebas paramétricas y no paramétricas, dependiendo de la distribución y naturaleza de las variables. Se compararon las diferencias en el promedio de las respuestas según sexo, tipo y lugar del consultorio, graduados en Chile o en el extranjero mediante el ANOVA. Se comparó Confianza en REUMA con las demás áreas mediante ANOVA de medición repetida. Los contrastes univariados, post hoc, se efectuaron mediante la prueba t de Student. 


\section{Tabla 1. Parámetros incluidos en la encuesta de evaluación de fortalezas, debilidades y Confianza de médicos de atención primaria en el abordaje de enfermedades reumatológicas}

1. Datos demográficos e información general.

1.1 Datos demográficos: Edad, sexo, universidad, nacionalidad, años de médico, tiempo en atención primaria, consultorio rural o urbano.

1.2 Interés personal en atención primaria.

¿Desea permanecer como MAP en el futuro o quiere una especialidad? ¿Cuál?

2. Evaluación de fortalezas y debilidades en el abordaje de REUMA.

2.1 Propios del MAP.

2.1.1 Interés personal en REUMA.

2.1.2 Calidad de formación de pregrado en REUMA.

2.1.3 Acceso a cursos de educación médica continua en REUMA.

2.2 Propias del consultorio.

2.2.1 Disponibilidad de literatura médica sobre REUMA en el consultorio.

2.2.2 Disponibilidad de exámenes de laboratorio complementario en el consultorio.

2.3 Propias de la organización del consultorio.

2.3.1 Posibilidad de intercambiar conocimientos con pares en el consultorio.

2.3.2 Acceso de los pacientes al reumatólogo desde el consultorio.

3. Evaluación de Confianza.

3.1 Confianza en habilidad para realizar una anamnesis y un examen físico. Cardiovascular, gastrointestinal, neurológico, respiratorio, músculo esquelético.

3.2 Confianza en abordaje clínico general (manejo) de enfermedades: cardíacas, endocrinas, gastrointestinales, hematológicas, infecciosas, nefrológicas, neurológicas, psiquiátricas, reumatológicas, respiratorias, del adulto mayor.

3.3 Confianza en conocimientos, diagnóstico y abordaje terapéutico de REUMA clásicas. Artrosis, lumbago, cervicalgia, hombro doloroso, RPB (tendinitis, bursitis, fibromialgia), osteoporosis, pie doloroso, AR, LES, SS, ESP, PM/DM y vasculitis.

3.4 Comparación de Confianza en abordaje terapéutico de enfermedades frecuentes: artrosis, diabetes mellitus, hipertensión arterial.

3.5 Confianza en capacidad para realizar procedimientos reumatológicos. Punción de rodilla y punción de hombro.

AR: artritis reumatoidea. Confianza: grado de confianza. ESP: esclerosis sistémica progresiva. LES: lupus eritematoso sistémico. MAP: médico de atención primaria. PM/DM: polimiositis/dermatomiositis. REUMA: enfermedades reumatológicas. RPB: reumatismos de partes blandas. SS: síndrome de Sjögren.

Cuando las variables no presentaron distribución normal se efectuó el test U de Mann-Whitney. Las asociaciones globales entre variables se estudiaron mediante regresión múltiple, seguidas por contrastes bivariados. Si la distribución no era normal se utilizó la prueba $\mathrm{R}$ de Spearman. Se calculó el tamaño muestral para detectar una diferencia promedio de 1 punto entre los grupos en comparación, asumiendo una DE de 3 puntos, error beta 0,2, error alfa 0,05 (diferencia estadísticamente significativa) y poder de $80 \%$, corrigiendo para prueba múltiple de hipótesis, según fuera el caso.

\section{RESULTADOS}

Se enviaron 763 encuestas, de las cuales, 347 fueron respondidas $(45,5 \%)$ (Tabla 2$)$.

Universidad de origen. Universidad de Chile 31\%, alguna Universidad de Ecuador 22\%, Universidad de la Frontera 13\%, Universidad de Cuba 7\%, Universidad de Concepción 5\%, otra Universidad de América Latina 5\%, Universidad de Europa del Este 3\%, Universidad Católica 3\%, no especificó $10 \%$. Los extranjeros se concentraban en región 
Tabla 2. C aracterísticas demográficas de los M édicos de Atención Primaria que participaron en el estudio

Número

Edad, promedio (rango)

$35,1(25-75)$

Sexo: hombre/mujer/no responde (\%)

$58,7 / 36,8 / 4,5$

Años de título de médico (rango)

$8(1-50)$

Meses en atención primaria, promedio (rango)

$61,5(1-276)$

$\mathrm{n}$

Nacionalidad

Chilena

202

Ecuatoriana

79

Cubana

18

Peruana

4

Boliviana

4

Rusa

3

Otros Región

No expresa su nacionalidad

Metropolitana

IX región

Tipo de consultorio, número (\%)

Urbano

289

Rural

58

metropolitana y los de la Universidad de la Frontera en la IX región.

Intereses de los MAP. Ciento ochenta (52\%) deseaban permanecer como MAP, 118 (34\%) querían abandonar APS, $14 \%$ no sabía o no respondió. Especialidades preferidas: medicina interna 43 (36\%), cirugía 18 (15\%), pediatría 15 (13\%), obstetricia y ginecología $11(9 \%)$ y $31(26 \%)$ otras.

Evaluación de fortalezas y debilidades. Lo mejor evaluado (puntaje promedio $\pm \mathrm{DE}$ ) fue interés personal para atender pacientes con REUMA $(7,4 \pm 2,1)$ y formación de pregrado en REUMA $(7,0 \pm 1,6)$. Lo peor evaluado: acceso al reumatólogo $(3,8 \pm 3,0)$ y disponibilidad de literatura médica $(2,2 \pm 2,0)$ (Figura 1). Dificultades para acceder al reumatólogo: demora o distancia excesivas $(91,3 \%)$, ausencia de centro de referencia $(8,7 \%)$.

Los MAP de región metropolitana evaluaron mejor acceso a EMC que los de la IX región $(4,7 \pm 2,8$ versus $3,4 \pm 2,3)$ (p 0,001$)$. Los de consul- torios urbanos evaluaron mejor la posibilidad de intercambiar conocimiento con pares $(4,9 \pm 3,0$ versus $3,9 \pm 2,7)(p$ 0,04). Los extranjeros evaluaron mejor su interés personal en REUMA que los nacionales $(7,9 \pm 2,1$ versus $7,1 \pm 2,1)(p 0,001)$.

Evaluación de Confianza. Confianza en habilidad para realizar anamnesis y examen físico: los MAP evaluaron mejor Confianza para realizar anamnesis que examen físico en las 5 especialidades consultadas (p 0,049). Aparato respiratorio: 8,6 $\pm 1,1$ y 8,5 $\pm 1,1$; gastrointestinal: $8,4 \pm 1,1$ y $8,3 \pm 1,1$; cardiovascular: $8,0 \pm 1,4$ y $7,7 \pm 1,4$; neurológico: $7,1 \pm 1,7$ y

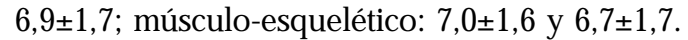

Confianza en el abordaje clínico general de enfermedades según especialidad: respiratorias: 8,1 $\pm 1,1$; gastrointestinales: $7,8 \pm 1,3$; del adulto mayor: 7,3 $\pm 1,6$; infecciosas: 7,1 $\pm 1,6$; cardiacas: $6,6 \pm 1,6$; reu-

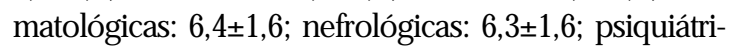
cas: $6,3 \pm 2,0$; endocrinas: $6,0 \pm 1,6$; neurológicas: $5,9 \pm 1,7$; hematológicas: $5,7 \pm 1,7$. No hubo diferencias según sexo, nacionalidad, tipo o lugar de consultorio. 


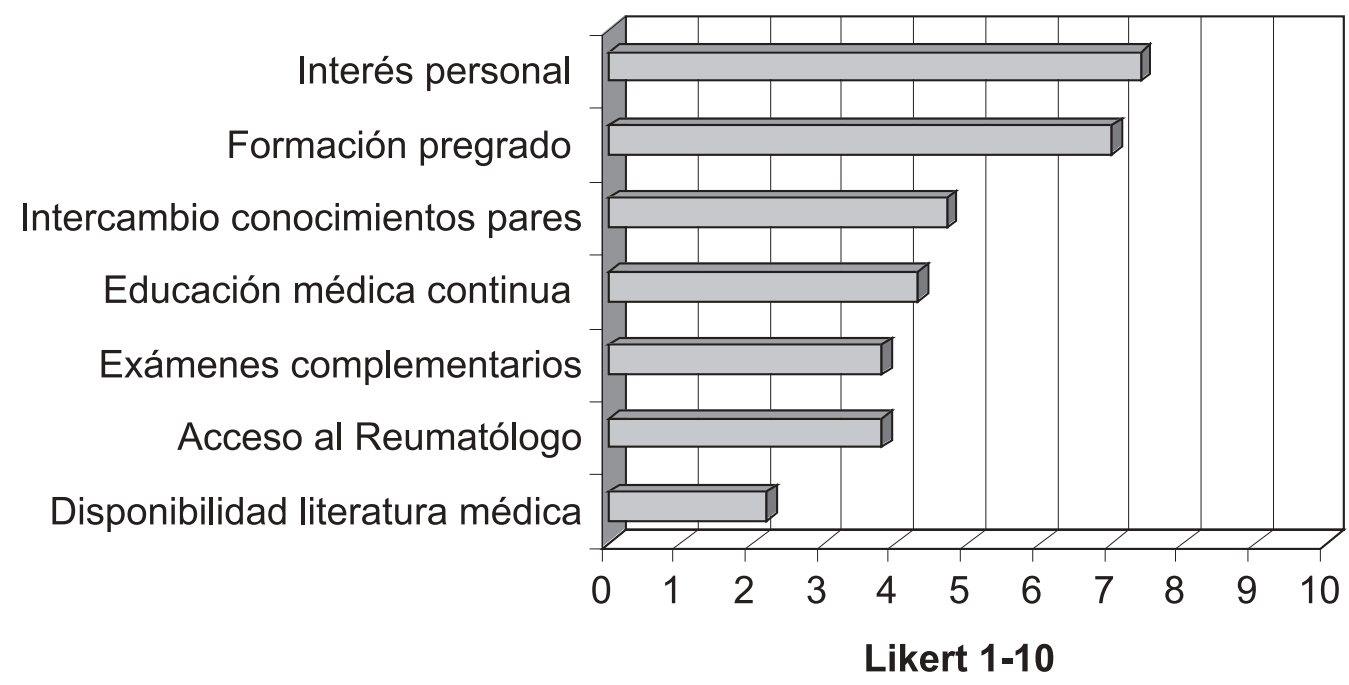

Figura 1. Autopercepción de fortalezas y debilidades de médicos de atención primaria en el abordaje de enfermedades reumatológicas.

Confianza en conocimientos, capacidad de hacer diagnósticos y abordaje terapéutico de REUMA clásicas: los MAP mostraron mayor Confianza en conocimientos y capacidad de hacer diagnósticos de REUMA no inflamatorias que inflamatorias (Figura 2). La Confianza en conocimientos $(6,7 \pm 1,5)$ fue mayor que Confianza en el abordaje terapéutico de REUMA $(6,1 \pm 1,5)$ ( $p$ 0,002). Los médicos extranjeros evaluaron mejor que los nacionales su Confianza en conocimientos $(7,1 \pm 1,4$ versus $6,6 \pm 1,5 ; \mathrm{p} 0,01)$ y abordaje terapéutico $(6,6 \pm 1,5$ versus $5,8 \pm 1,6$; $p$ 0,0007). No hubo diferencias según sexo, región o tipo de consultorio.

Una mejor evaluación de formación de pregrado o de EMC en reumatología se relacionó con mayor Confianza en conocimientos respecto a REUMA ( $p$ 0,02) pero no con Confianza en capacidad de hacer diagnósticos 0 abordaje terapéutico de REUMA (p 0,5 y 0,9, respectivamente).

Comparación de Confianza en abordaje terapéutico de enfermedades frecuentes: el puntaje fue mayor para HTA $(8,4 \pm 1,6)$ y diabetes mellitus $(7,7 \pm 1,7)$ que para artrosis $(7,3 \pm 1,7)$.

Confianza en la habilidad para realizar procedimientos reumatológicos: la Confianza en capacidad para realizar una punción de rodilla fue $4,7 \pm 3,0$ puntos y para una punción de hombro de 3,4 42,6 puntos. La Confianza para punción de rodilla fue mayor en los MAP de IX región respecto a los de la región metropolitana $(5,9 \pm 3,0$ versus $4,1 \pm 2,9$ ) (p 0,02 ). La Confianza en procedimiento fue inferior en mujeres que en hombres, punción de rodilla: $3,5 \pm 2,7$ versus $5,3 \pm 3,0(\mathrm{p} 0,0002)$ y punción de hombro $2,4 \pm 2,2$ versus $3,9 \pm 2,7$ (p 0,0002 ).

\section{DisCUSIÓN}

Este estudio fue diseñado para evaluar la autopercepción de fortalezas, debilidades y Confianza respecto a REUMA y otras enfermedades por MAP, con el objetivo primario de compararlos en términos relativos. Con el fin de incluir la mayor variedad de condiciones laborales, aspectos biodemográficos y de educación profesional, el estudio abarcó a todos los MAP de las regiones metropolitana y IX. Aun cuando la tasa global de respuesta fue de $45,5 \%$, el número de respuestas excedió en más del doble del tamaño muestral calculado. El impacto que las encuestas no respondidas pudiera tener en la solidez y validez de este estudio no puede ser estimado directamente, pero cabe destacar que los MAP que 


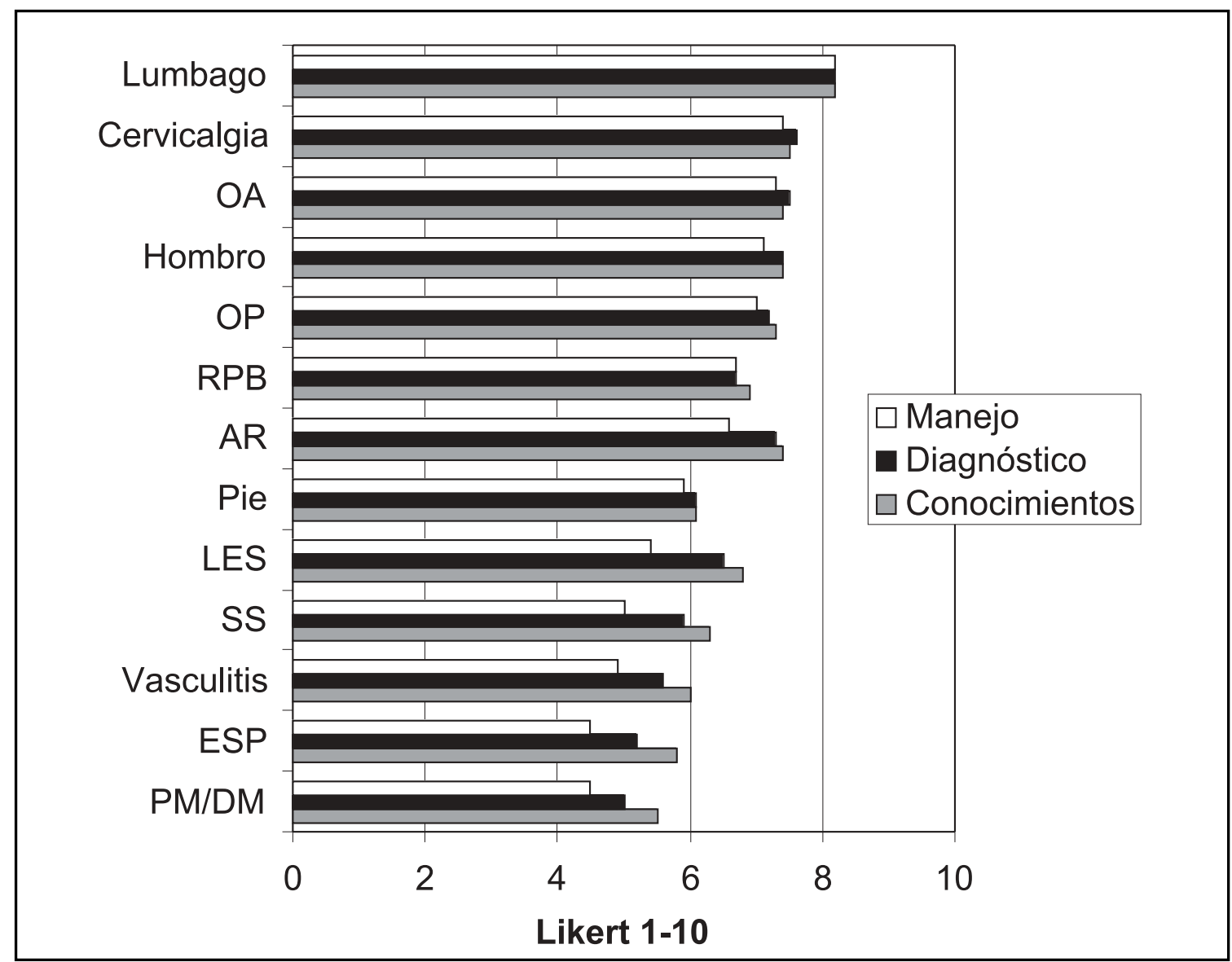

Figura 2. Confianza de médicos de atención primaria en conocimientos, capacidad para efectuar diagnósticos y abordaje terapéutico (manejo) de enfermedades reumatológicas. La confianza en conocimientos fue mayor que en abordaje terapéutico ( $\mathrm{p}$ 0,002). AR: artritis reumatoidea. Confianza: grado de confianza. LES: lupus eritematoso sistémico. OA: osteoartrosis. OP: osteoporosis. PM/DM: polimiositis/dermatomiositis. RPB: reumatismos de partes blandas. SS: síndrome de Sjögren. ESP: esclerosis sistémica progresiva.

respondieron las encuestas fueron comparables con aquellos que no las respondieron respecto de todas las características conocidas a priori (sexo, lugar de trabajo, región, nacionalidad). Por otra parte, las tasas de respuestas se situaron en los rangos esperados para este tipo de estudios. Como el objetivo primario fue evaluar autopercepción para enfrentar pacientes reumatológicos, más que determinar una prevalencia o un desempeño objetivo, esta tasa de respuestas es menos importante.

Las hospitalizaciones de causa reumatológica son poco habituales ${ }^{15}$, a diferencia de las consul- tas ambulatorias que son frecuentes tanto a nivel primario como secundario ${ }^{16}$. Este trabajo, realizado en APS, mostró que el perfil de los MAP era el de un grupo de mediana edad, preferentemente masculino, que permanecía en APS un tiempo promedio superior a 5 años y con un alto porcentaje de médicos extranjeros. Más de 50\% deseaba continuar en atención primaria en el futuro. Estos datos difieren del concepto clásico de MAP, encasillados como médicos que emigran rápidamente de esa condición. Por el contrario, emerge un grupo con interés de continuar en ella. 
Al evaluar la autopercepción de fortalezas y debilidades de APS para enfrentar REUMA, se observó que lo mejor valorado por los MAP fue su interés personal y su formación de pregrado en estas patologías, lo que puede considerarse como fortalezas. Esta evaluación fue mejor en los médicos extranjeros. Por el contrario, podemos considerar como debilidades el acceso a EMC en REUMA, que es peor evaluado por MAP de la IX región, junto a aspectos propios del consultorio, como disponibilidad de literatura médica, de exámenes complementarios y acceso al reumatólogo.

Respecto a Confianza, como fue medido en este estudio debe interpretarse como percepción de autoeficacia, la que a su vez ha mostrado ser un determinante de la conducta ${ }^{8,10}$. Es importante aclarar que una autoevaluación alta de Confianza no implica automáticamente mayor competencia clínica ${ }^{17}$. En algunos casos puede significar altos niveles de interés, como probablemente suceda con los médicos extranjeros que trabajan en APS. En cambio, los niveles bajos de Confianza se relacionan con mala formación, mal entrenamiento, poca experiencia o bajo interés y puede llevar a un manejo subóptimo lo que puede traducirse en incapacidad para hacer diagnósticos o realizarlos en forma tardía ${ }^{17}$. Una baja Confianza para realizar procedimientos puede significar que el médico se inhiba de efectuarlos, aunque esté indicado ${ }^{18,19}$.

En la autoevaluación de Confianza los MAP mostraron una amplia gama de respuestas. En habilidades, la Confianza para realizar una anamnesis y examen físico músculo-esquelético fue la peor evaluada, resultando inferior que el sistema neurológico considerado universalmente como el más complejo. Lo mejor evaluado fue el sistema respiratorio y gastrointestinal, lo que coincidió con mayor Confianza en el abordaje de esas enfermedades.

La Confianza en REUMA se evaluó en tres aspectos: conocimientos, diagnóstico y abordaje terapéutico; el resultado global mostró que los MAP tenían mayor confianza en sus conocimientos que en el abordaje terapéutico de REUMA. La Confianza en los tres parámetros estudiados fue mejor en los reumatismos no inflamatorios, lo que es esperable ${ }^{20}$. Sin embargo, si comparamos la artrosis con HTA y diabetes mellitus, todas enfermedades de gran prevalencia, observamos que los MAP tenían menor Confianza en el abordaje de la primera. La Confianza en reumatismos inflamato- rios como $A R$ y LES fue menor. Aun cuando existen discrepancias sobre el rol de los MAP en los reumatismos inflamatorios, se sabe que el pronóstico de estas enfermedades depende de su tratamiento precoz, por lo que la sospecha diagnóstica oportuna y la derivación precoz son de crucial importancia. En Chile, se ha demostrado un retardo promedio de 4 años antes que un paciente con AR acceda a un reumatólogo ${ }^{21}$, en otros países hay datos similares ${ }^{22,23}$.

Lo peor evaluado fue Confianza para realizar procedimientos simples que son de gran importancia diagnóstica y terapéutica, como punción de rodilla u hombro.

La formación en reumatología tiene dificultades en pregrado y postítulo. El pregrado, se desarrolla preferentemente en unidades de nivel terciario con pacientes y enfermedades distintas a las habituales en APS. Pese a la prevalencia de REUMA, se ha descrito que en pregrado se prefiere profundizar en otras especialidades ${ }^{7}$. En postítulo, el MAP no tiene contacto con docentes para continuar su entrenamiento, salvo en los cursos de EMC. Por otro lado, el reumatólogo no tiene experiencia en APS, por lo que probablemente los objetivos de la EMC diseñados por ellos no satisfagan las necesidades de los MAP, lo que es un requisito para una EMC efectiva $^{24,25}$. Estas dificultades del pregrado y postítulo en reumatología podrían explicar la baja Confianza de los MAP en hacer una anamnesis y examen físico reumatológico; para realizar diagnósticos y procedimientos y para abordaje terapéutico de los REUMA, a pesar de que tengan Confianza en sus conocimientos.

En resumen, los MAP evaluaron bien su interés en REUMA, mal su interacción con EMC y reportaron baja Confianza en sus habilidades clínicas en reumatología. Para mejorar esto parece necesario potenciar la EMC, especialmente en regiones. La EMC debería orientarse preferentemente al entrenamiento en destrezas y abordaje terapéutico de enfermedades ${ }^{7}$. Los cursos de EMC en reumatología pueden incluir una mezcla de técnicas de aprendizaje ${ }^{26,28}$. Las autoridades de salud deberían incentivar esa capacitación y aportar material de estudio en los consultorios. Los reumatólogos deberían preocuparse de la formación y entrenamiento del MAP tal como se preocupan del diagnóstico, tratamiento y rehabilitación de los enfermos de su especialidad ${ }^{29}$. 


\section{REFERENCIAS}

1 Targets for Health for All. Copenhagen: WHO Regional Office for Europe. 1985.

2. Reginato A. Reumatología para médicos de atención primaria. En: Pacheco D y Radrigán F, ed. REUMA. Santiago: Tangram Ediciones. 2002; 3-4.

3. Zamora L, Areliano M, Kuntsmann S, Montenegro A, Riveros B, Schegel et al. Invalidez en sistema privado de pensiones en Chile. Rev Méd Chile 1997; 125: 99-106.

4. Rosemblatt R, Cherkin D, Schneeweis R. The structure and content of family practice: Current status and future trends. J Fam Pract 1982; 15: 681-722.

5. Felts W, Yeun E. The economic impact of the rheumatic diseases in the United States. J Rheumatol 1989; 16: 867-84.

6. Pacheco D, Vizcarra G, Castilo V, Fuentealba C, Alvarez M, Ballesteros F. Perfil de la consulta reumatológica. Comparación entre un policlínico de atención primaria vs uno de reumatología. Revista Chilena de Reumatología 1997; 13: 101.

7. BJELE A. Primary care and rheumatology in musculoskeletal disorders: Bridging the gap. J Rheumatol 1996; 23: 205-7.

8. Glazier R, Dalby D, Badiey E, Hawker G, Bell M. Determinants of physician confidence in the primary care management of musculoskeletal disorders. J Rheumatology 1996; 23: 351-6.

9. BANDURA A. Self-efficacy: Toward a unifying theory of behavior change. Psychol Rev 1977; 84: 191-215.

10. HoLDEN G. The relationship of self-efficacy appraisal to subsequent health related outcomes: A metaanalysis. Soc Work Health Care 1991; 16: 53-91.

11. Streiner D, Norman G. Health measurements scales. A practical guide to their development and use. $2^{-}$ Edition. New York: Oxford University Press. 1995.

12. The Health Communication Unit at the Centre of Health Promotion. University of Toronto. Conducting Survey Research, 20 Edition, 1999; 1-60.

13. Woodward CA, Chambers LW. Guide to questionnaire construction and question writing. Parkdale, Canadian Public Health Association, 1982.

14. FeINSTEIN AR. In: Clinimetrics. Feinstein AR, Editor. Yale University Press. 1987. Nomenclature and functional classification of clinimetric indexes pp 6-21.

15. Pacheco D, Alvarez Me, Vizcarra G, Fuentealba C, Marinovic MA, BaLuesteros F. Características de la hospitalización reumatológica en un hospital de nivel terciario. Rev Méd Chile 2001; 129: 653-9.

16. Badiey E, Rasooly I, WeBSTER G. Relative importance of musculoskeletal disorders as a cause of chronic health problems, disability and health care utilization. J Rheumatology 1994; 21: 505-14.

17. Marteau T, Johnson M, Wynne G, Kaye W, Evans T. Cognitive factors in the explanation of the mismatch between confidence and competence in performing basic support. Psychology and Health 1989; 3: 173-82.

18. Salem-Schatz S, Avorn J, Soumerai S. Influence of clinical knowledge, organizational context, and practice style on transfusion decision making. Implications for practice change strategies. JAMA 1990; 264: 471-5.

19. Marteau T, Wynne G, Kaye W, Evans T. Resuscitation: Experience without feedback increase confidence but not skill. BMJ 1990; 300: 849-50.

20. Roberts C, Adebajo A, Long S. Improving the quality of care of musculoskeletal condictions in primary care. Rheumatology 2003; 42: 703-4.

21. Kaliski S, Bustos L, Artigas C, Alarcón C, Vega M, CÁRDENAS C. Artritis reumatoidea en población mapuche. Una experiencia de 16 años en la IX región de Chile. Rev Méd Chile 2001; 129: 253-8.

22. Hanly J, McGregor A, Biack C, Bresnihan B. Late referral of patients with rheumatoid arthritis to rheumatologists. Ir J Med 1984; 153: 316-8.

23. KIDd B, CaWley M. Delay in diagnosis of spondarthritis. Br J Rheumatol 1988; 27: 230-2.

24. HOCHBINDER R, BEL M. Critical appraisal of continuing medical education in rheumatic diseases for primary care physicians. Arthritis Rheum 1995; 38: 533-8.

25. Renner B, Devews B, Ennest S, Friedman C, Hoyle $\mathrm{R}, \mathrm{C}$ Rowell W et AL. Clinical rheumatology training of primary care physicians: the residente perspective. J Rheumatology 1990; 17: 666-72.

26. Brown A, Clarke S, Coady D, Haq I. Educational research fellowships. Rheumatology 2004; 543-6.

27. Davis P, Russell A, Skeith K. The use of standardized patients in performance of a needs assesment and development of a CME intervention in rheumatology for primary care physicians. Rheumatology 1997; 24: 1995-9.

28. Murphy M, Biack N, Lamping D. Consensus development methods, and their use in clinical guideline development. Health Technol Asses 1998; 2: 3-7.

29. LEWTAS J. OMERED outcome measures in rheumatology education. J Rheumatology 1996; 23: 208-10. 